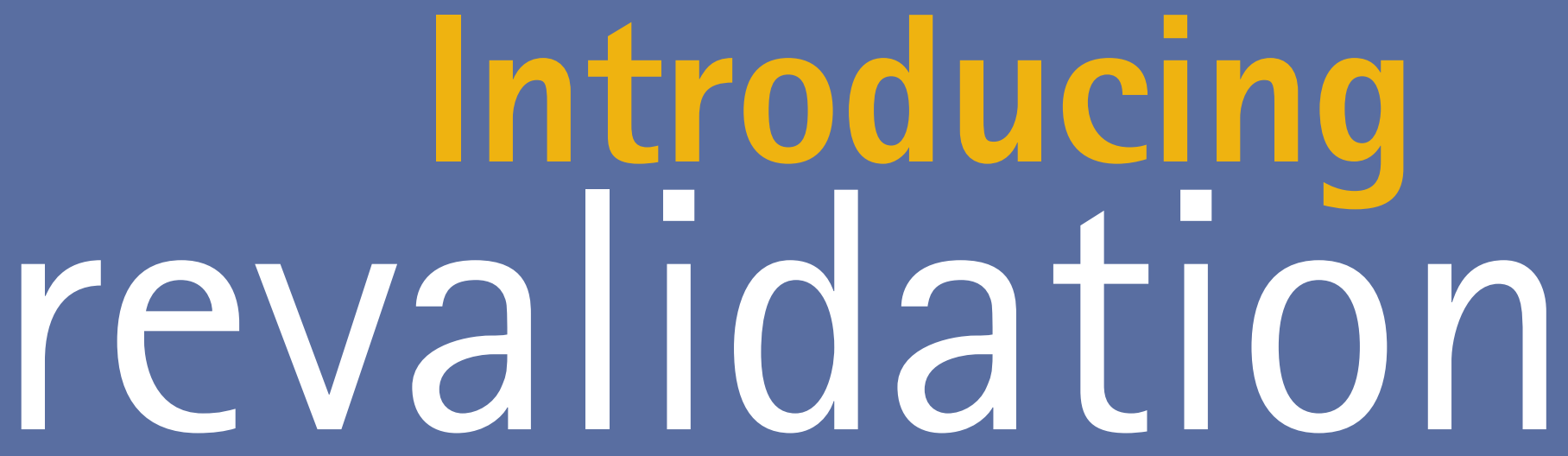

Continuing education in dentistry is always a hot topic and the GDC's announcement of the introduction of a process of revalidation for dentists has increased the temperature even further. In this article, the GDC answer some key questions about revalidation, what it means for you as a dentist, what will be involved and when it will begin.

\section{What does 'revalidation' mean?}

As a registered dentist or dental care professional, you know that you have to keep your knowledge up-to-date to stay on the GDC register. You do this already by carrying out continuing professional development (CPD). But keeping up-to-date through CPD is only one aspect of showing that the standard of your practice is up to the level required by the GDC and expected by your patients. That is why in future all our registrants will need to be able to show that they continue to meet our standards to stay on the register.

Revalidation will be based on a set of standards, against which we will ask you to collect evidence. The standards will be focused on four headings:

- Professionalism

- Clinical

- Communication

- Management and leadership.

A three stage process is proposed, as shown in Figure 1.

\section{Why is the GDC}

introducing revalidation?

Patients need to have confidence that the professionals providing their dental care have not just shown that they meet our standards when they join our registers, but can show that they continue to meet the standards expected of them over the course of their working lives.

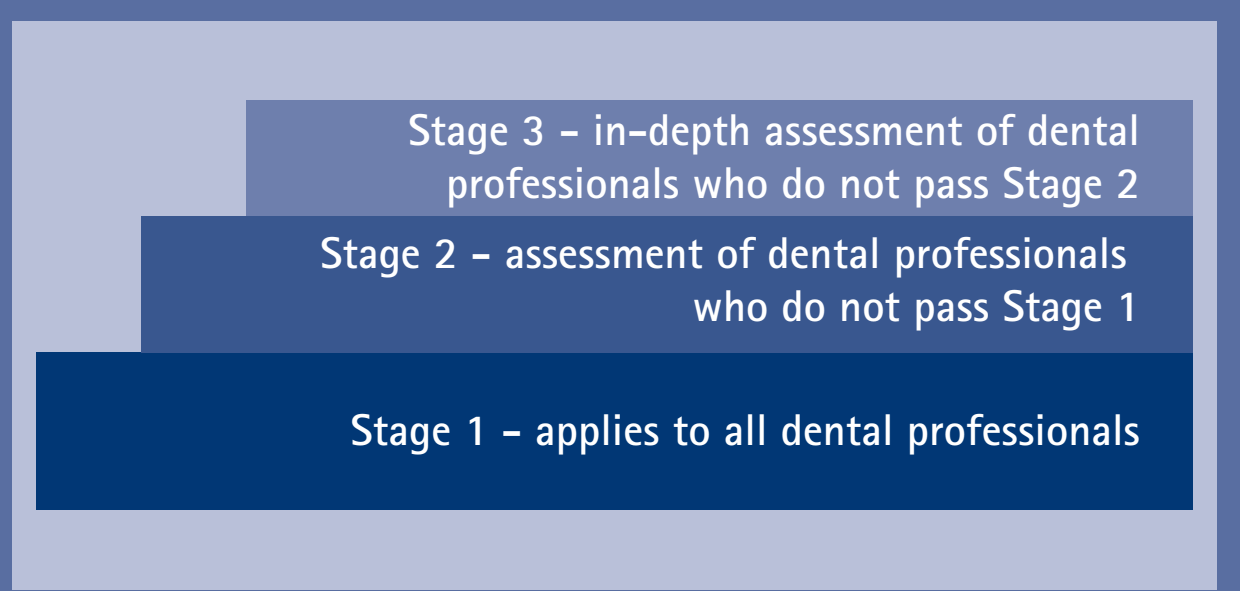

Fig. 1 Schematic of the three proposed stages of the revalidation process

This does not mean that dental professionals who already do a lot of work to make sure that their performance is up-to-scratch will be bombarded with a lot of unnecessary paperwork. We want revalidation to be as simple and flexible as possible, and we are committed to making use of existing and forthcoming quality assurance systems and locally gathered evidence wherever available and appropriate. This could include, for example, material you collect as part of a good practice scheme or practice development planning process.

We are going to pilot the proposals below extensively to see if they work. This means that they are not 'set in stone', and you have the opportunity to make suggestions on what revalidation should include and how it should work. We will be holding an open consultation on our proposals from September onwards. Please take the opportunity to look at the consultation on our website and let us know your suggestions and views.

\section{What does revalidation mean in} practice? Will I need to keep some sort of portfolio of evidence?

You will be required to produce a portfolio of evidence for Stage 1 of the process. We are developing a standards and evidence framework for revalidation (more information about this is available on our website at www.gdc-uk.org). The types of evidence that you might provide could include (but might not be limited to) any of the following: 
- Clinical audit/peer review

- Patient satisfaction surveys

- Personal and practice development plans

- Evidence of CPD compliance

- Anonymised patient records

- Practice accreditation schemes

- Significant event analysis

- Appraisal.

The framework will be flexible so that you can select the type of evidence you provide for each of the standards from a menu of options. At the end of your revalidation cycle, you will need to submit a declaration of the evidence you have to show that you meet our standards for continued registration. This would determine whether you pass the threshold for revalidation (we expect that most of our registrants would pass the threshold at this stage). Stage 1 is intended to be a sift to determine who requires a 'further look'. It is not a guarantee that those not put through to Stage 2 are fit to practise; we can never 'guarantee' this.

\section{What if I don't (or can't) provide adequate evidence?}

We are working with professional bodies to make sure that our requirements for revalidation are not unnecessarily time consuming and that they make use of evidence that you already have access to wherever possible. In fact, you may already have all the information that we will be looking for. We expect that (as with CPD at the moment) there will be a small minority of you who do not comply with the requirements to produce evidence. If you simply do not comply, you risk removal from the register. If you try to comply, but struggle to do so, we will refer you into Stage 2.

Stage 2 may involve peer assessment in the practice against a check list, examining patient records, medical histories, radiographs and so on. A comprehensive check list will be developed for this purpose. At this stage, if you do not appear to meet the standards required, you may be allowed additional time (perhaps working with a mentor) to bring your performance up to standard. If you are unable to do that (or there are serious concerns about your performance from the assessment) you will have to go into Stage 3 of our process.

At Stage 3, we expect that there would be a menu of assessment routes and tools available, but you would have to show through a robust assessment process that you met our requirements to remain on the register.
If you could not do so, you would not be allowed to continue practising. We would hope to see as few as possible dental professionals in Stage 3, as it is much better for you and your patients if you seek help from colleagues and professional bodies at an early stage if you think that you are having difficulties, rather than avoiding problems until the last minute.

\section{Will my patients be asked to rate me} as part of the revalidation process?

Yes, the evidence you provide might include patient assessment questionnaires or other forms of multi-source feedback. There will be a number of other types of evidence that you can collect within your portfolio. If you would like to offer any suggestions on what we should be looking for, please let us know.

\section{When will revalidation start?}

We expect the first revalidation cycle to begin for some dentists in 2011. There will much more consultation and discussion between now and then, so look out for regular updates as we develop our plans.

\section{Who will it apply to? Will it be the same for everyone?}

Ultimately revalidation will apply to all registered dentists and dental care professionals, but our work so far has concentrated on dentists in general dental practice in the first instance. Everyone will have to provide evidence that they continue to meet a set of standards under the four domains. Most of these will be common to everyone, but as we develop them we will consider whether some should be tailored to your own individual type of practice.

What are your plans for people who don't work in general dental practice?

We are looking at ways in which individuals who work outside the general practice setting, for example, dental professionals working in a teaching role, can meet the requirements for revalidation. We would be keen to hear your views. We are also looking at how specialists will revalidate in their specialties, and we would welcome views on this as well.

\section{Is there anything I can be doing now} to gear up for revalidation?

Yes, you may be doing plenty already! You can use personal and/or practice development plans to highlight areas of development that would keep your standards up to date and benefit your patients (and get into the habit of keeping evidence of what you are doing). You might also consider joining a good practice scheme.

\section{How will the piloting work?}

We will be piloting Stage 1 of the process with groups of dentists in 2009. We want to work with them to establish what the time commitment might be for the process, whether the process we have outlined will work in practice and if not, how it needs to be changed, and whether we are asking for the right kind of information. This feedback will help to develop the final version of our standards and evidence framework for revalidation.

\section{Can I take part in a pilot?}

There are going to be three pilot sites and you may be able to take part if you are in one of the areas. Please contact us on revalidation@gdc-uk.org if you would like more information. We would be interested in hearing your views even if you are not taking part in a pilot.

\section{Is all the detail worked out already or can I have a say on it?}

Yes, you can have a say and we would welcome your views. The revalidation section of our website includes a summary of our current proposals in more detail - we are looking for feedback, so please have a look and send us your comments. There is a first draft of the standards and evidence framework that we are proposing for Stage 1 of the revalidation process - we would particularly welcome your thoughts on how we can develop this into a final workable tool for dental professionals. That part of the website will be regularly updated and we welcome input at any time - please email us on revalidation@gdc-uk.org.h

DOI: 10.1038/sj.bdj.2008.743 